\title{
Solving linear Neumann boundary value problems using block methods
}

\begin{abstract}
A direct two-point block one-step method for solving linear Neumann boundary value problems (LNBVP) is considered. This method will solve the second order LNBVP directly without reducing it to the system of first order equations. The direct solution of LNBVP will be calculated at two points simultaneously using constant step size. This method will be used together with the linear shooting technique to construct the numerical solution. The implementation is based on the predictor and corrector formulas in the $\mathrm{PE}(\mathrm{CE}) \mathrm{m}$ mode. Numerical results are given to show the performance of this method compared to the existing methods.
\end{abstract}

Keyword: Block method; Neumann boundary value problems 